\title{
On-tissue derivatization of lipopolysaccharide for detection of lipid A using MALDI-MSI
}

Hyojik Yang ${ }^{1}$, Courtney E. Chandler ${ }^{1, \wedge}$, Shelley N. Jackson ${ }^{2}$, Amina S. Woods $^{2,3}$, David R. Goodlett ${ }^{1,4}$, Robert K. Ernst ${ }^{\#, 1}$, Alison J. Scott ${ }^{\#, 1,5 *}$

${ }^{1}$ Department of Microbial Pathogenesis, School of Dentistry, University of Maryland, Baltimore, MD 21201 USA

2 Structural Biology Core, NIDA IRP, NIH, 333 Cassell Drive, Room 1120, Baltimore, MD 21224, USA

${ }^{3}$ Pharmacology and Molecular Sciences, Johns Hopkins University School of Medicine. Baltimore, MD 21205 USA

${ }^{4}$ University of Gdansk, International Centre for Cancer Vaccine Science, Gdansk, Poland, EU

${ }^{5}$ Maastricht Multimodal Molecular Imaging (M4I) Institute, Maastricht University, Maastricht, Netherlands, EU

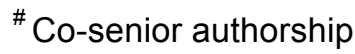

Corresponding Author Information

Alison J. Scott

650 W. Baltimore St - 8 South

Baltimore, MD 21201

(410) 706-6112

ascott1@umaryland.edu 


\section{SUPPLEMENTAL INFORMATION CONTENTS}

LLA-MSI Process Scheme

S-3

Additional Figures \& Data

S4-S8

Expanded Materials \& Methods

S9-S11

Expanded Results \& Discussion

S12-S13

Expanded References

S14 


\section{On-tissue acid hydrolysis process}

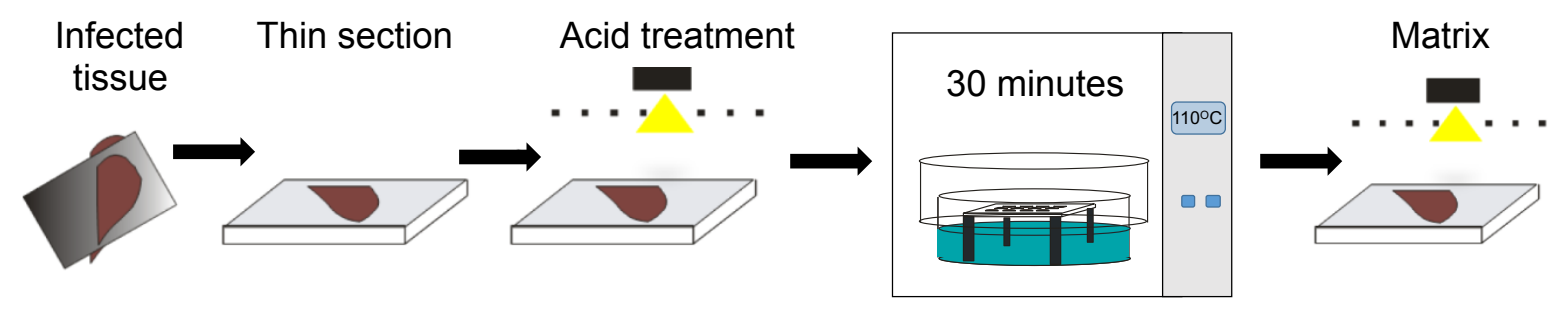

\section{Acid hydrolysis of LPS to Lipid A}

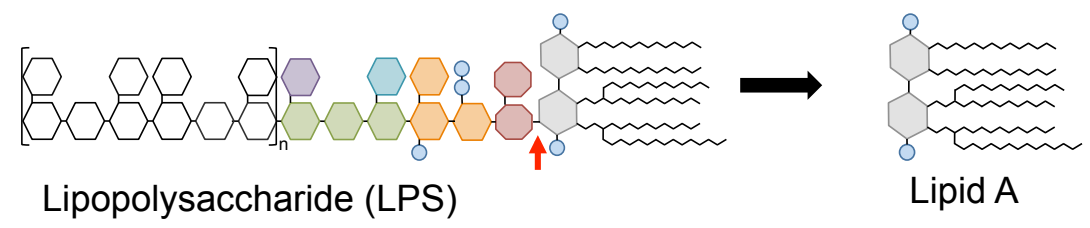

Scheme 1. Procedure of derivatization of smooth LPS on-surface or on-tissue using liquid sprayer. (top) After heating at $110^{\circ} \mathrm{C}$ for 30 minutes samples were analyzed by MALDI-TOF MS. Full-length LPS is acid hydrolyzed by the process in the top panel to lipid A (red arrow), which is the minimal component of LPS retaining endotoxic activity. (bottom) 

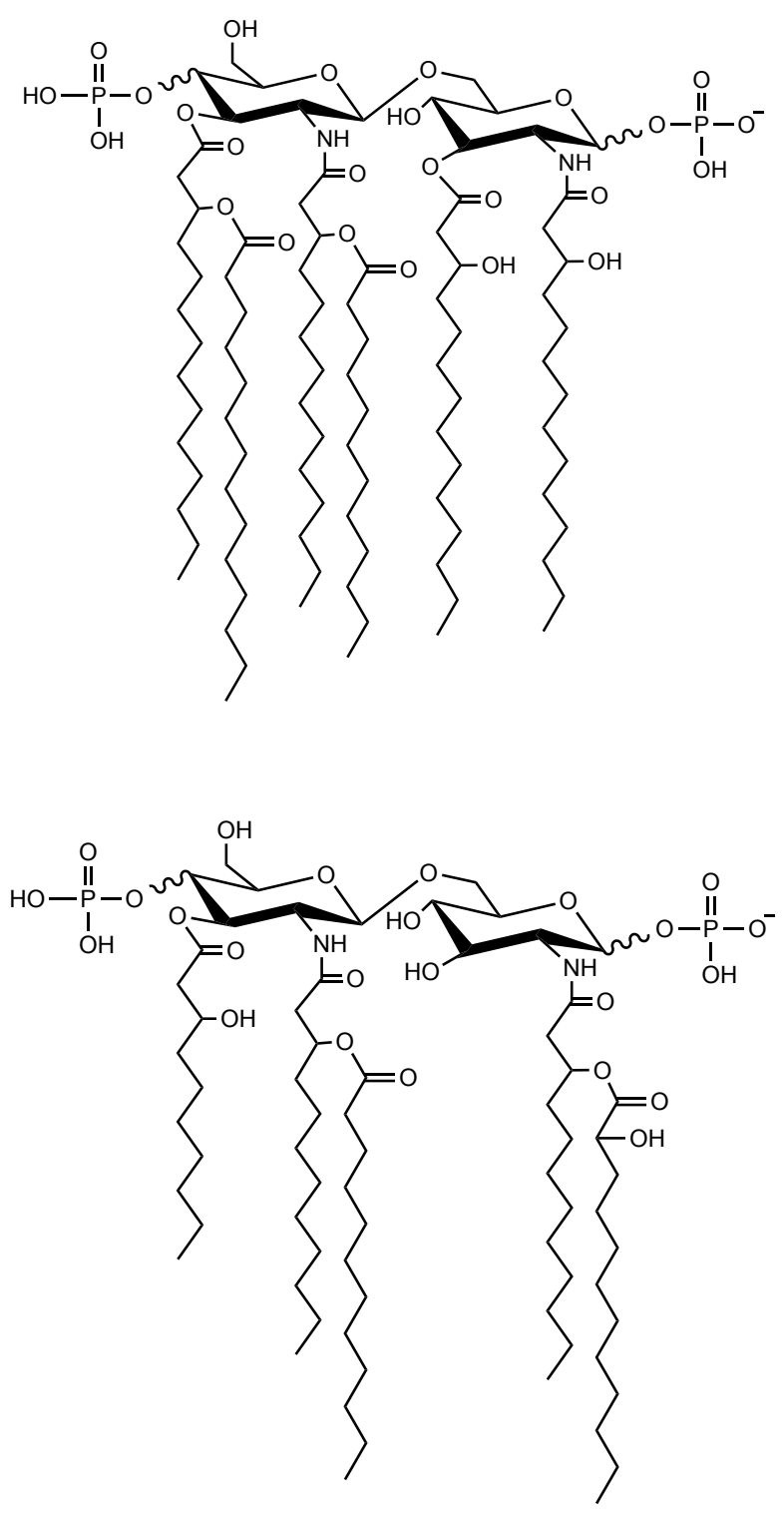

Figure S1. Major lipid A structures referenced in this study. Deprotonated lipid A corresponding to the ions $\mathrm{m} / \mathrm{z} 1797$ (top) E. coli and $\mathrm{m} / \mathrm{z} 1446$ (bottom) $P$. aeruginosa. 


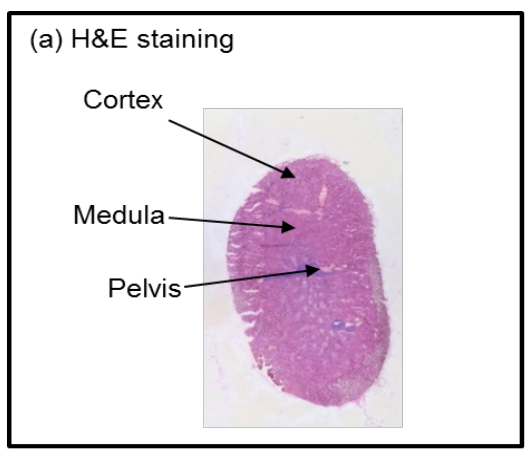

(d) $\mathrm{m} / \mathrm{z} 496.1 \pm 0.5$

(5)

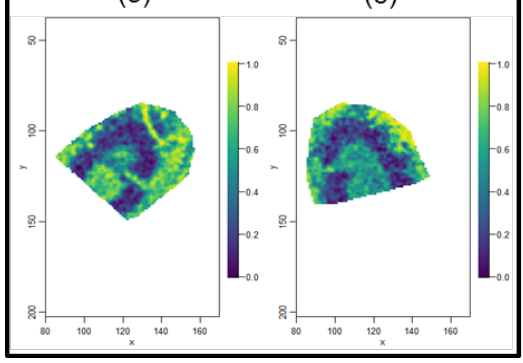

(b) $\mathrm{m} / \mathrm{z} 878.4 \pm 0.5$

(1)

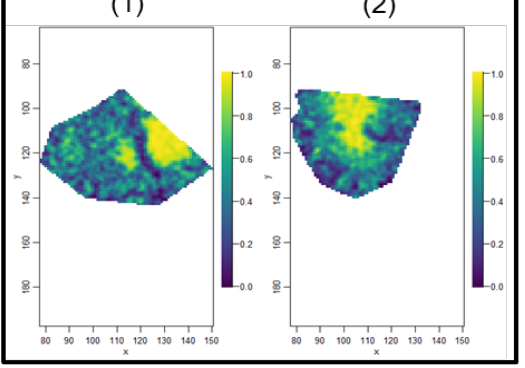

(e) $\mathrm{m} / \mathrm{z} 703.2 \pm 0.5$

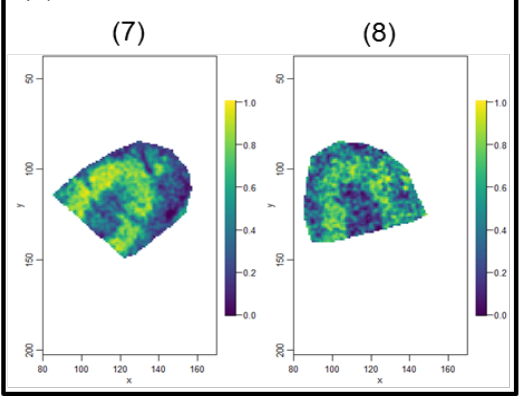

(c) $\mathrm{m} / \mathrm{z} 885.4 \pm 0.5$

(3)

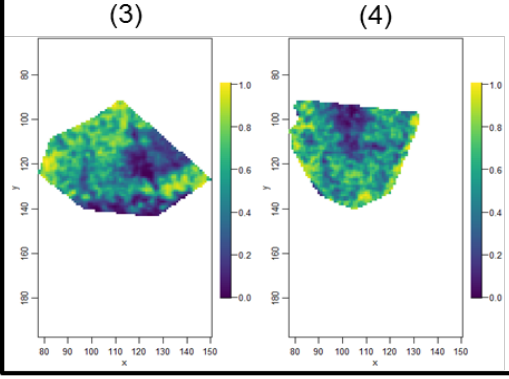

(f) $m / z 760.2 \pm 0.5$

(9)

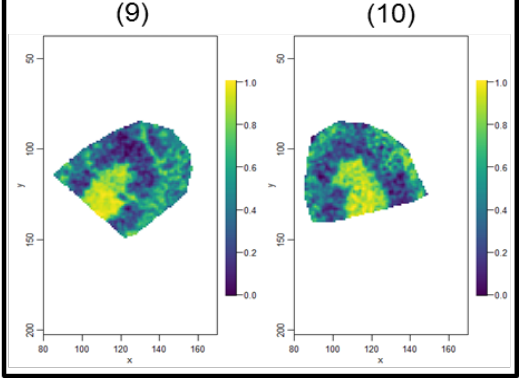

Figure S2. MALDI-MSI data of kidney tissue before and after heat treatment. (a) H\&E staining image of the mouse kidney. Ion images of $\mathrm{m} / \mathrm{z} 878.4 \pm 0.5$ (b) and $885.4 \pm 0.5$ (c), negative ion mode. Ions at $\mathrm{m} / \mathrm{z} 496.1 \pm 0.5$ (d), $703.2 \pm 0.5$ (e), and $760.2 \pm 0.5$ (f) were collected from positive ion mode. (b-f) Mouse kidney sections, untreated tissue (left, 1) and heat-treated tissue (right, 2) compared for relative ion intensity, MALDI-TOF MSI, normalized (TIC), raster width $100 \mu \mathrm{m}$. 
E. coli $\mathrm{m} / \mathrm{z} 1797$
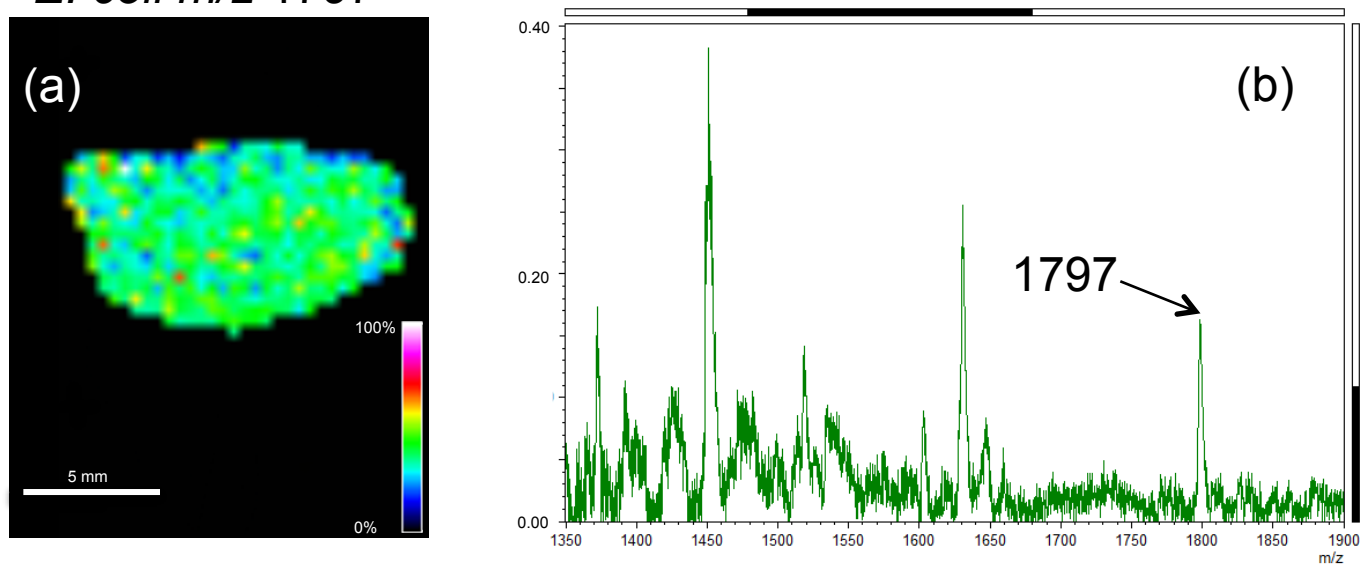

$P$. aeruginosa $\mathrm{m} / \mathrm{z} 1446$
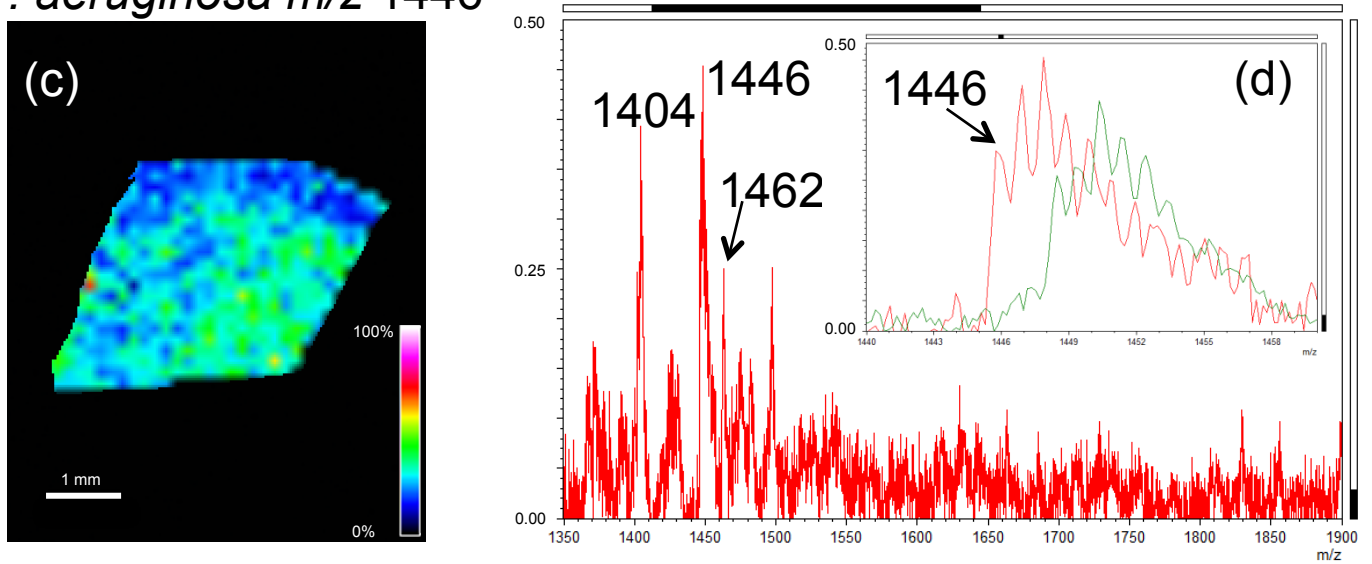

Figure S3. Lipid A ion maps from lung loaded with bacteria suspended in gelatin inflation solution. Ec- (a) Pa- (c) inflated mouse lung evaluated by LLA-MSI showing $\mathrm{m} / \mathrm{z} 1797$ and 1446, respectively. Averaged MALDI-MSI spectra of $E c(b)$ and $P a(d)$, respectively. The inset on the figure (d) is overlaying Ec spectrum (b, green) and $P a$ spectrum (d, red) in $\mathrm{m} / \mathrm{z}$ region from 1440 to 1460 . (a-d) MALDI-TOF MSI, normalized (TIC), raster width 400 and $100 \mu \mathrm{m}$ for (a) and (c), respectively. 

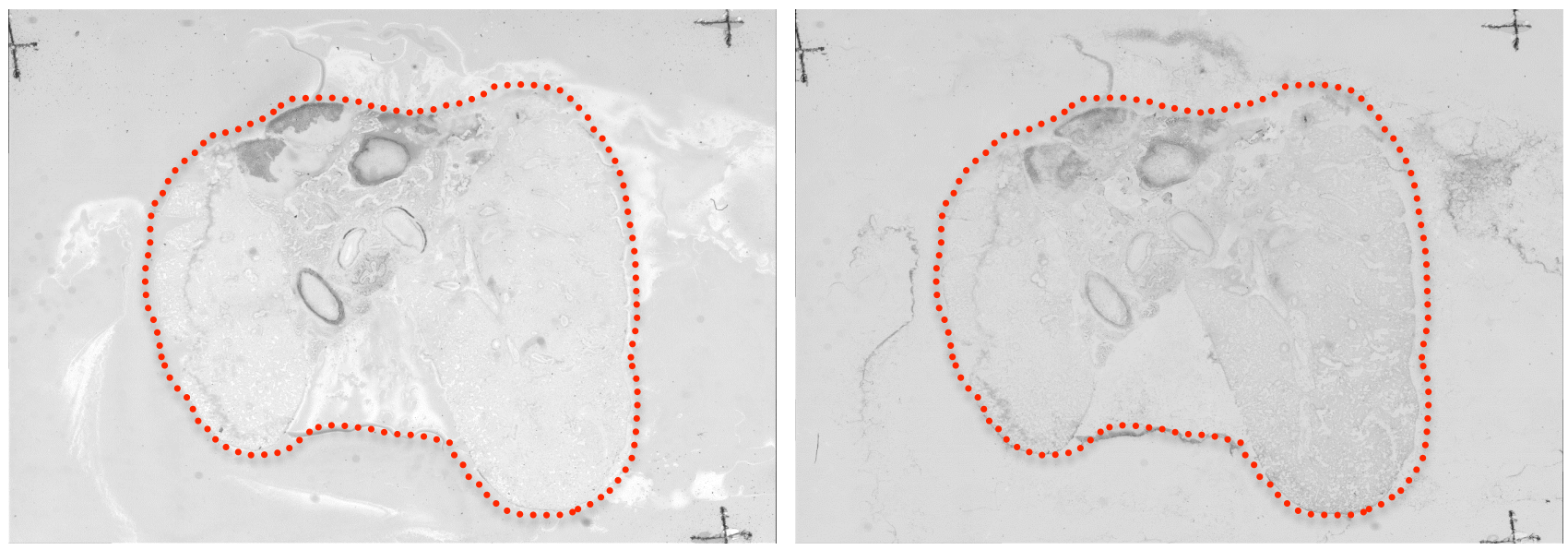

Figure S4. Absence of overt section damage following LLA-MSI treatment process. Light scanning image of inflated lung tissue before (left), and after (right) heat treatment of the tissue for preparation of data in Figure S3. Red dots outline the tissue borders. 


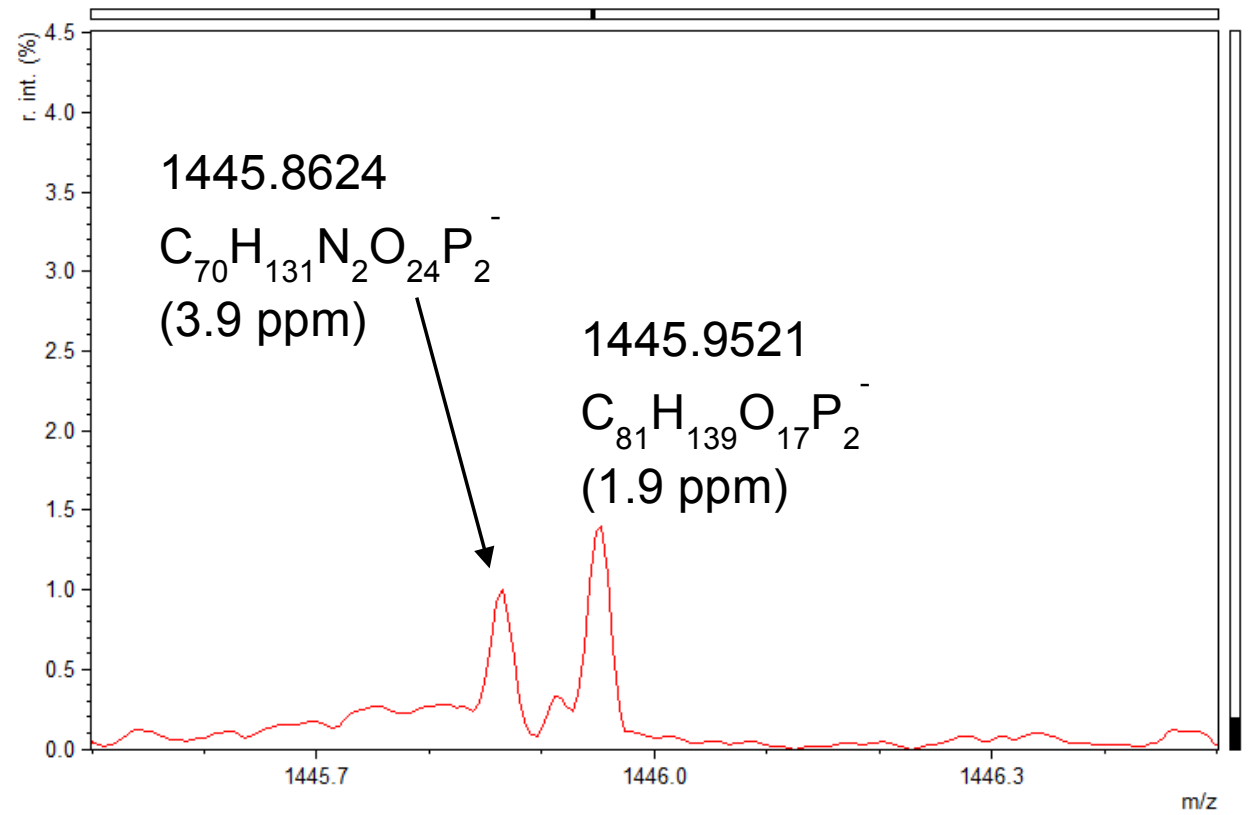

Figure S5. Averaged mass spectrum showing robust signal for $P$ a lipid $A$ with the derivatization chemistry. The thickness of tissue section is $25 \mu \mathrm{m}$. Spectrum showing $\mathrm{Pa}$ lipid $A$ ion $(m / z 1445.8624)$ when the derivatization chemistry is applied, clearly separated from neighboring cardiolipin peak ( $\mathrm{m} / \mathrm{z}$ 1445.9521). Chemical formulas are given for both and $\mathrm{Pa}$ lipid A and cardiolipin with respective mass errors in parentheses. MALDI-IT-Orbitrap MSI. 


\section{EXPANDED MATERIALS \& METHODS}

\section{Materials}

Methanol (MeOH, 98\%), norharmane (NRM, 98\%), chloroform (HPLC grade), isobutyric acid, ammonium hydroxide, porcine gelatin, trisodium citrate, and citric acid were purchased from Sigma-Aldrich (St. Louis, MO, USA). Phosphate-buffered saline (PBS) and certified endotoxinfree water were sourced from Gibco (Grand Island, NY, USA). Indium tin oxide (ITO) float glass slides were sourced from Delta Technologies (Loveland, CO, USA). Focus MALDI plates were obtained from Hudson Surface Technology (Suwon, South Korea). Powdered nutrient media and agar (as listed below) for bacterial cultures were purchased from Beckton-Dickinson (Hunt Valley, MD, USA) and magnesium chloride supplement was from Sigma-Aldrich (St. Louis, MO, USA). Agilent tuning mix was used for instrument calibration (Santa Clara, CA, USA). Female C57BL/6 adult mice were used for these studies and all work was approved by the Institutional Animal Care and Use Committee of the University of Maryland, Baltimore.

\section{Bacterial strains and growth conditions}

Two bacterial species producing LPS were used to evaluate the on-tissue acid hydrolysis method: Ec ATCC 25922 and Pa ATCC 31482 (sourced from the American Type Culture Collection - ATCC, Manassas, VA). Ec and Pa were grown in lysogeny broth (LB) supplemented with $1 \mathrm{mM}$ magnesium chloride in shaking liquid culture (200 RPM) to mid-log phase, with an optical density $\left(\mathrm{OD}_{600}\right)$ value given for each microbial suspension per experiment. Growth on solid media (plates) consisted of the same LB formulation with the addition of $1.5 \%(\mathrm{w} / \mathrm{v})$ Bacto-

agar. For lipid A microextraction development on-tissue, $1 \mathrm{~mL}$ of liquid culture was harvested by centrifugation (8000 × g, $5 \mathrm{~min})$ and supernatant aspirated. Bacterial pellets were resuspended 
in PBS and turbidity was measured using a Thermo Scientific Biomate $3 S$ UV-Vis spectrophotometer (Waltham, MA, USA).

\section{Data collection and instrumentation}

Mass Spectrometer: MALDI-TOF MS analyses were performed on a Bruker Daltonics Autoflex Speed mass spectrometer (Billerica, MA, USA) equipped with a Nd:YAG (355 nm wavelength; 3 ns pulse width) SmartBeam laser. The laser power energy was adjusted between $0 \%$ and $100 \%$ to provide the laser pulse energy between 93.8 and $121.8 \mu \mathrm{J}$. A typical laser pulse energy of $20 \%$ (about $99.4 \mu \mathrm{J}$ ) was applied in the experiments, except where otherwise stated. A MTP 384 target from Bruker Daltonics was used for sample plate. The instrument was operated in linear mode with accelerating voltage, grid voltage and delayed extraction time set to $19 \mathrm{kV}, 90 \%$, and 120 ns, respectively. Unless otherwise stated, each mass spectrum was acquired as an average of 500 laser shots at $10.0 \mathrm{~Hz}$ frequency. A MALDI-TOF mass spectrometer Microflex LRF interfaced with FlexImaging Software from Bruker Daltonics (Billerica, MA, USA) was operated in the reflectron mode. The instrument is equipped with a standard nitrogen laser with $337 \mathrm{~nm}$ wavelength and a maximum repetition rate of $20 \mathrm{~Hz}$. Standard instrument parameters were used, although delayed extraction times were optimized to between 400 and $800 \mathrm{~ns}$. To perform imaging of tissue specimens in a Microflex instrument without introducing modifications of either the laser beam or molecular ions path distances, we loaded tissues on the back side of single-use stainless steel target plates (inverted onto magnetic holder) (0.3T non-hydrophobic microflex target, Hudson Surface Technology, Suwon, South Korea). Both instruments were calibrated with Agilent tuning mix (for lipid A experiments) or red phosphorous cluster negative ions (phospholipid experiments). Ec and Pa lipid A on-tissue were assigned using accurate mass determination by HR-MSI on a MALDI LTQ-XL-Orbitrap (Thermo Fisher, San Jose, CA). Xcaliber and ImageQuest software were used for MALDI-MSI data acquisition and processing. 
Image acquisition, normalization, and visualization were carried out using flexImaging 4.1, and Cardinal $\mathrm{MSI}^{1}$ software and spectral analysis with mMass software (version 5.5.0).

\section{SAFETY STATEMENT}

No unique chemical hazards are presented in this work that warrant extra precautions outside of the typical safe handling and protective equipment necessary to handle low $\mathrm{pH}$ solutions and heat sources. Bacteria should be handled according to their assigned biosafety category using all the normal and respective precautions thereof. 


\section{EXPANDED RESULTS \& DISCUSSION}

\section{Tissue integrity and spatial preservation of lipids following LLA-MSI}

Since this procedure included heating at $110^{\circ} \mathrm{C}$ for 30 minutes in a humidified chamber, we sought to evaluate the effect on tissue integrity with respect to both overall visual damage and delocalization of ions of interest. Other histological methods including immunohistochemistry procedures commonly include an antigen retrieval step using wet heat to recover antigen conformation using various instruments such as microwave or high-pressure cookers. ${ }^{2,3}$ To evaluate tissue integrity following the LLA-MSI method we compared the scanning image of a gelatin inflated mouse lung (13 $\mu \mathrm{m}$ tissue thickness) prior to and after the incubation and washing steps. No major losses of tissue were noted from these images (Figure S4). However, maintaining tissue integrity does not prevent delocalization of molecules on the tissue. We chose mouse kidney sections because it is a widely accepted benchmark validation tissue and kidney sections show good segregation of endogenous lipids in the different tissue compartments (cortex, medulla, and pelvis). ${ }^{4}$ Figure S2a demonstrates the distribution of these three regions as an H\&E stained image of the tissue used for MSI (Figure S2b-f). Figure S2b demonstrates a sulfatide $(18: 1 / 22: 02 \mathrm{OH})$ ion at $\mathrm{m} / \mathrm{z} 878.4 \pm 0.5$ and the distribution on tissue before (1) and after (2) heat treatment. The odd numbers and even numbers throughout Figure $\mathbf{S 2}$ represent before and after heat treatment, respectively. The ion is mainly localized in the kidney pelvis even after heat treatment with comparable before and after ion maps. The ion at m/z $885.4 \pm 0.5$ (phosphatidylinositol 38:4) in Figure S2c showed a distribution mainly observed in the medullar and cortex regions both before and after treatment. This result was similar to those found in previous studies. In positive ion mode, results show the same trend as negative ion mode, illustrated in the ion distribution at $\mathrm{m} / \mathrm{z} 496.1 \pm 0.5$ (putatively identified as LysoPC 16:0) (Figure S2c) that exhibits similar mapping localized in the cortex and pelvis regions 
before (5) and after (6) heat treatment and signal is absent in the medullary region. In contrast to LysoPC, sphingomyelin (SM 34:1) was putatively identified at $\mathrm{m} / \mathrm{z} 703.2 \pm 0.5$ and the corresponding ion image is shown in Figure S2d. SM34:1 is predominantly distributed in the medulla in both cases (7) and (8), respectively, before and after heat treatment. High abundance was observed within the pelvis region for the ion window $\mathrm{m} / \mathrm{z} 760.2 \pm 0.5$ (putatively identified as phosphatidylcholine, 34:1) before and after treatment in both (9) and (10) as shown in (Figure S2e). Of note, the ions demonstrated in Figure $\mathbf{S 2}$ are known to contain multiple lipid isobars and isomers and the corresponding maps are demonstrated for comparison purposes with the major known constituent of the peak serving as the putative identity. The ion distributions we interrogated in mouse kidney showed no appreciable change in localization or abundance even after heat treatment. Further, our results show no tissue deterioration after heat treatment which means the LLA-MSI method can be used to study host-pathogen interactions by observing both lipid A and mammalian endogenous lipids, simultaneously. 


\section{REFERENCES}

(1) Bemis, K. D.; Harry, A.; Eberlin, L. S.; Ferreira, C.; Ven, S. M. van de; Mallick, P.; Stolowitz, M.; Vitek, O. Cardinal: An R Package for Statistical Analysis of Mass Spectrometry-Based Imaging Experiments. Bioinform Oxf Engl 2015, 31 (14), 2418-2420.

https://doi.org/10.1093/bioinformatics/btv146.

(2) Shi, S.-R.; Cote, R. J.; Taylor, C. R. Antigen Retrieval Techniques. J Histochem Cytochem 2001, 49 (8), 931-937. https://doi.org/10.1177/002215540104900801.

(3) R, V. K.; Jones, D.; Udupa, V. A Simple and Effective Heat Induced Antigen Retrieval Method. Methods 2016, 3, 315-319. https://doi.org/10.1016/j.mex.2016.04.001.

(4) Muller, L.; Kailas, A.; Jackson, S. N.; Roux, A.; Barbacci, D. C.; Schultz, J. A.; Balaban, C. D.; Woods, A. S. Lipid Imaging within the Normal Rat Kidney Using Silver Nanoparticles by MatrixAssisted Laser Desorption/Ionization Mass Spectrometry. Kidney Int 2015, 88 (1), 186-192. https://doi.org/10.1038/ki.2015.3. 